\title{
STUDY OF SACCADIC EYE MOVEMENTS IN DIAGNOSTIC IMAGING
}

\author{
Jianxun Lou ${ }^{1}$, Xin Zhao ${ }^{1}$, Philippa Young ${ }^{2}$, Richard White ${ }^{3}$, Hantao Liu ${ }^{1}$ \\ ${ }^{1}$ School of Computer Science and Informatics, Cardiff University, Cardiff, United Kingdom \\ ${ }^{2}$ Breast Test Wales, National Health Service, Cardiff, United Kingdom \\ ${ }^{3}$ Department of Radiology, University Hospital of Wales, Cardiff, United Kingdom
}

\begin{abstract}
Eye movements reflect the visual process of humans' perception and cognition. In the field of medical imaging, the diagnosis rendered by radiologists is closely related to their eye movements when reading radiological images. It is beneficial to study the eye movements of radiologists to improve the diagnostic performance. However, existing studies are mainly focused on the radiologists' fixations but rarely on their saccade patterns. Moreover, these studies are almost based on limited datasets. In this paper, we present a quantitative study of the gaze behavior of radiologists from the perspective of saccade patterns on a large-scale dataset. The dataset comprises of the eye-tracking data of 10 expert radiologists reading 196 mammograms. By analyzing the saccade amplitude, direction, and bias of radiologists, we found that radiologists have specific saccade patterns in image reading and the saccade patterns are significantly affected by the different reading phases, working experience, and orientations of the mammograms.
\end{abstract}

Index Terms - saccade, eye movement, medical imaging, eye-tracking, mammogram

\section{INTRODUCTION}

Humans tend to concentrate their visual resources on the most relevant information in a visual scene. Eye movements represent a central feature of human vision, which reflects the visual system processes of perception and cognition [1]. It has been demonstrated that radiologists' diagnosis is related to eye movement behavior when reading radiological images [2] [3]. According to past studies, the miss rate (i.e., meaning discrepancies in terms of missing an important diagnosis) might be up to 30\% [4] and the false-positive rate may even exceed 50\% [5] in some areas of radiological diagnostic practice. Therefore, it is beneficial to study radiologists' eye movement behavior during the image reading, and develop useful solutions to improve their diagnostic performance.

Generally, eye movements are composed of fixations (the stages that the eyes are almost still to receive information) and saccades (the eye movements between the current and next fixation) [6]. In the field of medical imaging, research attention is especially focused on fixations. For example, a method is proposed in [7] to use the radiologists' fixation features (i.e., fixation count, duration, and frequency) to evaluate their diagnostic performance. In [8], the experts' fixation patterns are utilized to train trainees and successfully improve their technical laparoscopic skills.

However, the saccades, which represent another key characterization of eye movements, are rarely analyzed in detail in the field of medical imaging. There is evidence to indicate that the direction and amplitude of saccadic eye movements are the pivotal features to describe gaze behavior. In [9] and [10], the overall average saccade amplitude in Computed Tomography images has been discussed. [11] and [12] show that the saccade is influenced by the context of images and the characteristics of readers. [13] found that the patterns in saccade direction vary with picture orientation. Therefore, it is of interest to investigate the saccade patterns of expert radiologists using eye-tracking technology and gaze data analysis.

In this paper, a novel saccade pattern study in screening mammography is proposed with the following contributions. First, this study is based on an eye-tracking dataset of a large number of mammogram images and expert radiologists which are often limited in existing studies. Second, the saccade amplitude and direction are utilized to characterize gaze behavior instead of the popular fixation-based metrics. Third, the saccade behavior differences between the different reading phases are revealed in this paper, which could be useful for the development of algorithms for automatically assisting radiologists in diagnosis.

\section{EYE-TRACKING MAMMOGRAPHY DATASET}

The dataset, as detailed in [14], contains eye-tracking data of expert radiologists reading a set of mammograms, which records the positions and start and end times of each fixation for each radiologist. This mammogram set consists of 196 mediolateral oblique (MLO) view mammogram images with the size of $1080 \times 1920$ pixels, which were extracted from 98 anonymous cases from the University Hospitals KU Leuven in Belgium, each with two MLO views for the left and right breasts respectively. Examples of the mammogram images in this dataset are shown in Fig.1. Eye-tracking data were col- 
lected from the mammogram reading processes of 10 expert radiologists from the Breast Test Wales (BTW) Cardiff center which is one of the centers delivering the National Health Service Breast Screening Programme (NHSBSP) that provides a screening mammography exam for women aged 50-70 every three years. These ten radiologists are denoted as being R1 (8 years experience in mammography), R2 (6 years), R3 (8 years), R4 (2 years), R5 (5 years), R6 (10 years), R7 (20 years), R8 (25 years), R9 (15 years), R10 (25 years). The procedure of collecting and processing the eye-tracking data for this dataset is detailed in [15].

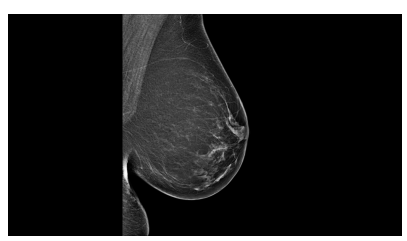

(a) Left Breast

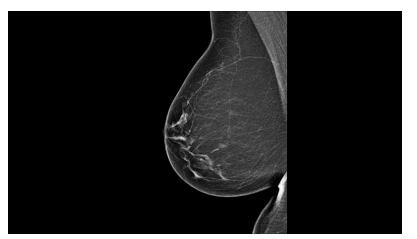

(b) Right Breast
Fig. 1. Illustration of two mammogram images in the dataset. (a) and (b) are respectively the MLO view mammogram images of the left and right breasts from one anonymous case.

\section{ANALYSIS OF THE SACCADE BEHAVIOR}

To clearly describe the pattern of saccade amplitude and direction of radiologists, the following conditions are given. The amplitude is defined as the Euclidean distance between the current and previous fixation; the direction is defined as the angle formed by the line between the current and previous fixation and the rightward horizontal line through the previous fixation; the amplitude and direction are both calculated from the second fixation of each image case.

\subsection{Analysis of the Saccade Amplitude}

The mean saccade amplitude over all images for each radiologist is shown in Fig. 2 (a). It can be seen that the mean saccade amplitude of the 'senior' radiologists with at least 15 years of experience in mammography (i.e., R7, R8, R9, and R10) is lower than the 'junior' radiologists with not more than 10 years of experience (i.e., R1, R2, R3, R4, R5, and R6). The result of the independent sample t-test shows there is a significant difference between the 'senior' and 'junior' groups $(\mathrm{p}<0.05)$, meaning 'senior' radiologists make significantly smaller saccade amplitudes than 'junior' radiologists. Furthermore, there are also relationships between the saccade amplitude and the reading periods of mammograms. It can be seen from Fig. 2 (b) and (c) that the mean amplitude of both groups is significantly larger between 0 and $500 \mathrm{~ms}$ than in other periods. After the $0-500 \mathrm{~ms}$ phase, the 'junior' group's mean saccade amplitude drops to the area right above 200 pixels and maintains in the region for the rest of the reading phases; the saccade amplitude of the 'senior' group first drops to the area right above 200 pixels and then further decreases after the 500-1000ms phase and finally the mean amplitude is plateauing at a rather low mean amplitude (i.e., below 200 pixels) towards the end of the reading.

According to the studies of [16] and [17], reading methods of radiological images mainly include global reading and detailed reading, and the following inferences can be made. First, both 'senior' and 'junior' radiologists scan the images extensively with similar mean amplitude to obtain a holistic impression and determine the regions of interest during the initial reading period $(0-500 \mathrm{~ms})$. Second, all radiologists tend to scan with a smaller amplitude to check these regions of interest after the initial period, but the 'senior' radiologists tend to implement more meticulous reading strategies. Therefore, all radiologists' readings of mammograms can be divided into two phases: the global reading $(0-500 \mathrm{~ms})$ and detailed reading (500-3000ms).

\subsection{Analysis of the Saccade Direction}

The study in [13] shows that the direction of readers' saccade is strongly affected by the presentation orientations of natural images. However, the influences of the presentation orientations on the radiological images are rarely discussed. As shown in Fig.1, the mammograms of the left and right breasts of each patient are presented with different orientations to the radiologists during the collection of eye movement data. Therefore, mammograms are firstly divided into the left and right breast sets in the analysis for saccade direction to avoid possible interference.

To describe the overall pattern of saccade directions, all directions are divided into 32 sections of the central angle with $11.25^{\circ}$ each, which are sequentially distributed counterclockwise using the rightward horizontal direction (i.e., $0^{\circ}$ ) as the beginning of the first section. The saccade direction proportions for the 'senior', 'junior', and all radiologists of each image set in each section are then plotted in the polar plots, as shown in Fig.3. It can be seen that the proportion distributions of the 'senior' and 'junior' groups are quite similar and there is no statistically meaningful difference (i.e., $\mathrm{p}>0.05$ ) between them in both the right and left breast sets. Comparing Fig. 3 (a) and (b), both of them similarly have one upward and one downward peaks vertically. There is an obvious difference in the horizontal direction, where, the proportion distribution of the left breast image set has one leftward peak (around $180^{\circ}$ ) and the right breast image set has one rightward peak (around $0^{\circ}$ ) horizontally. This is consistent with the different orientations of these two sets of mammograms.

In order to conduct statistical analysis, all saccades in the aforementioned 32 sections are re-arranged into four directions that include upward $\left(45^{\circ}-135^{\circ}\right)$, leftward $\left(135^{\circ}-225^{\circ}\right)$, downward $\left(225^{\circ}-315^{\circ}\right)$, and rightward $\left(315^{\circ}-45^{\circ}\right)$ saccades. 


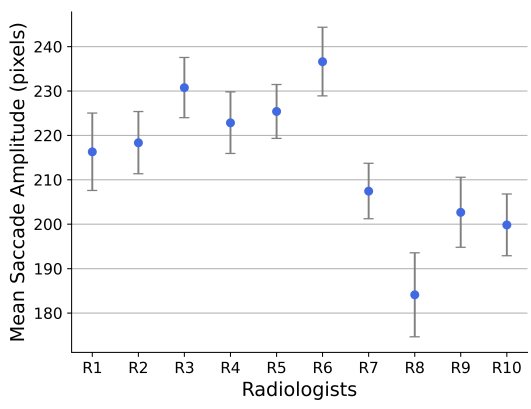

(a)

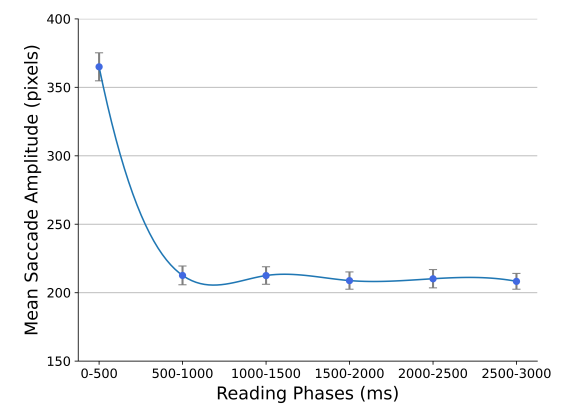

(b)

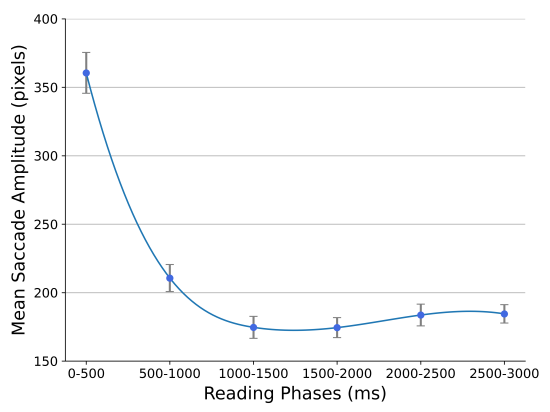

(c)

Fig. 2. Illustration of the mean saccade amplitude. (a) represents the individual mean amplitude. (b) and (c) show the mean saccade amplitude respectively for the 'junior' and 'senior' radiologists for different reading time periods. The error bars represent the $95 \%$ confidence interval.

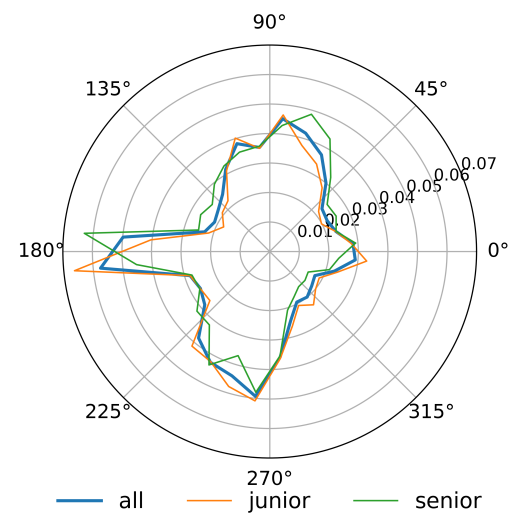

(a)

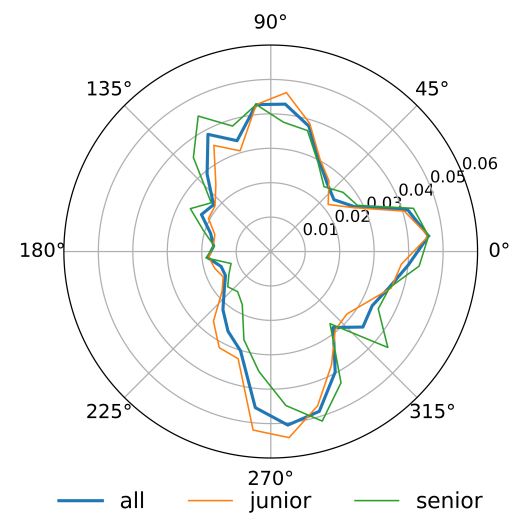

(b)

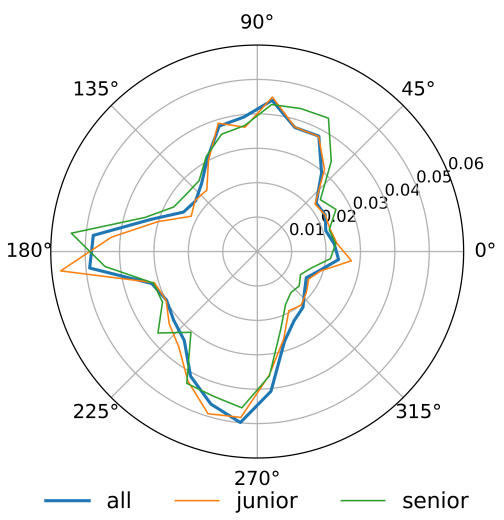

(c)

Fig. 3. Illustration of the proportions of saccade direction. (a) and (b) are respectively for the left and right breast image sets. (c) is for all mammograms after left aligning.

According to the results of the ANOVA performed for the four saccade directions, the effect of the different directions (i.e., $\mathrm{F}=3.86, \mathrm{p}<0.05$ ) on the saccade frequency is statistically significant. Besides, the paired t-test is performed for saccades of four directions in the left and right breast image sets. The results show that vertical (upward or downward) saccades there is no significant difference (i.e., $\mathrm{p}>0.5$ ) between the two image sets, but for the horizontal (leftward and rightward) saccades there is a significant difference (i.e., $\mathrm{p}<0.01)$ between the two image sets. Moreover, there is no significant difference $(\mathrm{p}>0.05)$ between the leftward saccades in the left breast image set and the rightward saccades in the right breast image set, and no difference between the rightward saccades in the left breast image set and the leftward saccades in the right breast image set. In summary, the following conclusions can be drawn. First, the saccade frequency is different in different directions. Second, only the horizontal saccade directions of radiologists are affected by the mammograms' orientations and this influence can be reduced by the orientation alignment.

Therefore, to reduce the influence of the different mammograms' orientations, all mammograms and data are aligned to the left, then a new overall saccade direction polar is shown in Fig.3 (c). Also, there is no significant difference (i.e., $\mathrm{p}>0.5$ ) between the 'senior' and 'junior' radiologists. It can be seen that radiologists tend to perform asymmetric horizontal saccades and more vertical direction saccades in the mammography reading. This is different from humans' behavior when viewing natural images, where humans are prone to scan symmetrically and scan heavily more in the horizontal direction [13] [18] [19]. It is speculated that these differences might be due to the radiologists' expertise, reading purpose, and specific image content.

\subsection{Analysis of the Saccade Bias}

In order to describe more clearly, the following conditions are given to estimate the saccade bias. First, all of the 98 right 


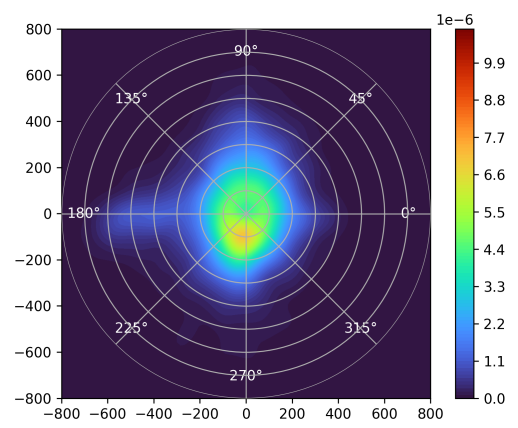

(a)

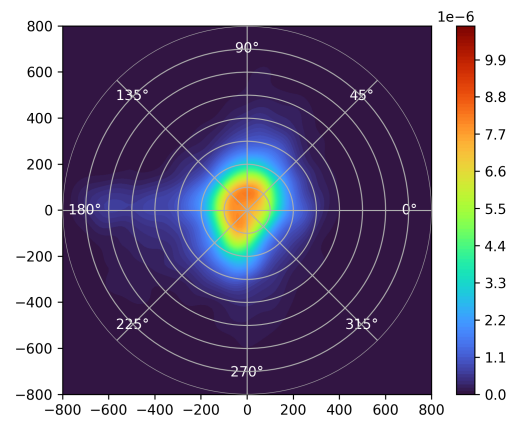

(d)

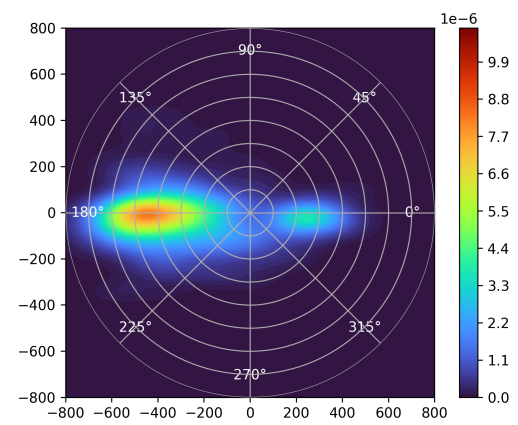

(b)

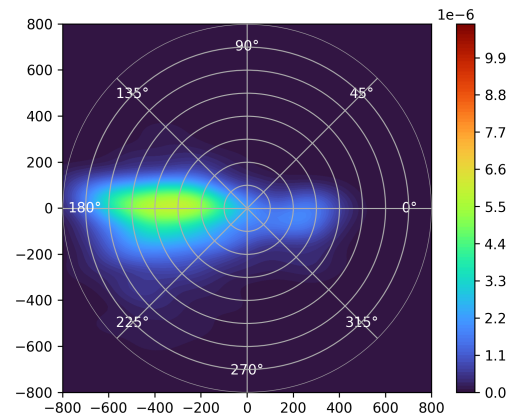

(e)

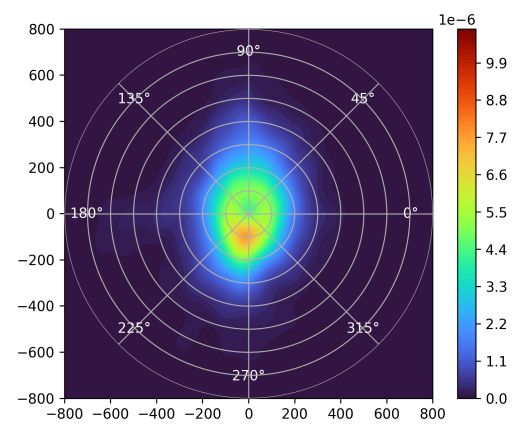

(c)

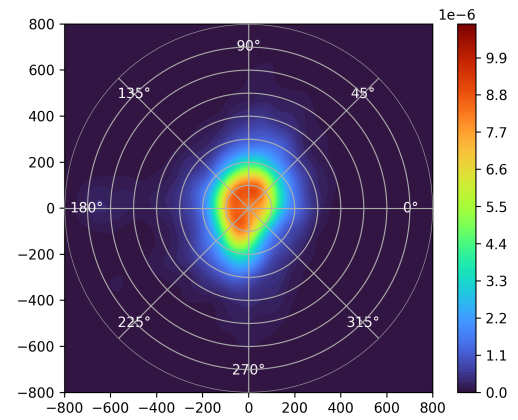

(f)

Fig. 4. Illustration of the saccade distributions of overall reading, global reading and detailed reading phases for junior radiologists (a), (b), (c) and senior radiologists (d), (e), (f).

breast images are left-aligned by horizontal flipping to reduce the presentation orientation influence that has been discussed above. Second, the maximum value of the saccade amplitude is limited to 800 pixels because there are rare saccade amplitudes larger than 800 pixels. Third, the probability distribution is estimated by the two-dimensional Gaussian kernel density estimation (KDE), which is expressed by:

$$
\widehat{f}_{(x, y)}=\frac{1}{n} \sum_{i=1}^{N} K_{h}\left(x-\Delta x_{i}, y-\Delta y_{i}\right)
$$

where $\mathrm{n}$ is the number of samples; $K_{h}$ is the scaled Gaussian kernel function; $\Delta x_{i}$ and $\Delta y_{i}$ respectively denote the horizontal and vertical components of the amplitude of each saccade.

It has been learned from the analysis of the saccade amplitude that the overall reading can be divided into global and detailed reading phases. Therefore, the saccade probability distributions of overall reading and the two reading phases of 'senior' and 'junior' groups over the entire 196 mammogram images are plotted separately in Fig.4. It can be seen from overall reading (see in Fig.4 (a) and (d)), the saccades of both 'senior' and 'junior' radiologists are mainly distributed in vertical and leftward horizontal directions and have a strong bias in the central area with amplitudes less than 200 pixels. In terms of detailed reading (see in Fig.4 (c) and (f)), the 'junior' group especially concentrates the saccades on the down- ward direction with amplitudes of about 50 to 200 pixels. In contrast, the concentration area of the 'senior' group is much larger, which indicates that the 'senior' radiologists are more inclined to conduct multi-directional small-scale saccades. This provides further evidence that the reading strategy of 'senior' radiologists is more meticulous. The saccade distributions of the detailed reading are vastly different from the global reading. In terms of the global reading (see in Fig.4 (c) and (f)), both groups have a strong horizontal saccade bias, but unlike with detailed reading, the saccades of 'junior' radiologists are more concentrated. This is probably because the 'senior' radiologists have developed more proficient searching skills that allow them to search more widely.

\section{CONCLUSION}

In this paper, we analyzed the image reading patterns from the perspective of the saccade for 10 expert radiologists based on a large-scale eye-tracking dataset. The analysis leads to the conclusion that radiologists have specific saccade patterns in mammogram image reading, which are these saccade patterns are significantly affected by different reading phases, degrees of mammography experience, and orientations of the mammograms. These could provide a basis for the development of algorithms that can automatically predict the diagnosis rendered by radiologists. 


\section{REFERENCES}

[1] E. Kowler, "Eye movements: The past 25years," Vision Research, vol. 51, no. 13, pp. 1457 - 1483, 2011.

[2] G. Tourassi, S. Voisin, V. Paquit, and E. Krupinski, "Investigating the link between radiologists' gaze, diagnostic decision, and image content," Journal of the American Medical Informatics Association, vol. 20, no. 6, pp. 1067-1075, 2013.

[3] E. A. Krupinski, "Visual scanning patterns of radiologists searching mammograms," Academic Radiology, vol. 3, no. 2, pp. 137-144, 1996.

[4] C. S. Lee, P. G. Nagy, S. J. Weaver, and D. E. NewmanToker, "Cognitive and system factors contributing to diagnostic errors in radiology," American Journal of Roentgenology, vol. 201, no. 3, pp. 611-617, 2013, Publisher: American Roentgen Ray Society.

[5] H. D. Nelson, M. Pappas, A. Cantor, J. Griffin, M. Daeges, and L. Humphrey, "Harms of breast cancer screening: Systematic review to update the 2009 u.s. preventive services task force recommendation," Annals of Internal Medicine, vol. 164, no. 4, pp. 256-267, 2016.

[6] K. Rayner, "The 35th sir frederick bartlett lecture: Eye movements and attention in reading, scene perception, and visual search," Quarterly Journal of Experimental Psychology, vol. 62, no. 8, pp. 1457-1506, 2009.

[7] L. McLaughlin, R. Bond, C. Hughes, J. McConnell, and S. McFadden, "Computing eye gaze metrics for the automatic assessment of radiographer performance during X-ray image interpretation," International Journal of Medical Informatics, vol. 105, pp. 11-21, 2017.

[8] S. J. Vine, R.S.W. Masters, J. S. McGrath, E. Bright, and M. R. Wilson, "Cheating experience: Guiding novices to adopt the gaze strategies of experts expedites the learning of technical laparoscopic skills," Surgery, vol. 152, no. 1, pp. 32-40, 2012.

[9] R. Bertram, J. Kaakinen, F. Bensch, L. Helle, E. Lantto, P. Niemi, and N. Lundbom, "Eye movements of radiologists reflect expertise in ct study interpretation: A potential tool to measure resident development," Radiology, vol. 281, no. 3, pp. 805-815, 2016.

[10] R. Bertram, L. Helle, J. K. Kaakinen, and E. Svedström, "The effect of expertise on eye movement behaviour in medical image perception," PLOS ONE, vol. 8, no. 6, pp. 1-15, 062013.

[11] O. Le Meur and A. Coutrot, "Introducing contextdependent and spatially-variant viewing biases in saccadic models," Vision Research, vol. 121, pp. 72-84, 2016.
[12] O. Le Meur, A. Coutrot, Z. Liu, P. Rämä, A. Roch, and A. Helo, "Visual attention saccadic models learn to emulate gaze patterns from childhood to adulthood," IEEE Transactions on Image Processing, vol. 26, no. 10, pp. 4777-4789, 102017.

[13] T. Foulsham, A. Kingstone, and G. Underwood, “Turning the world around: Patterns in saccade direction vary with picture orientation," Vision Research, vol. 48, no. 17, pp. 1777-1790, 2008.

[14] L. Lévêque, P. Young, and H. Liu, "Studying the gaze patterns of expert radiologists in screening mammography: A case study with breast test wales," in 28th European Signal Processing Conference (EUSIPCO), 08 2020.

[15] L. Lévêque, H. Bosmans, L. Cockmartin, and H. Liu, "State of the art: Eye-tracking studies in medical imaging," IEEE Access, vol. 6, pp. 37023-37034, 2018.

[16] H. L. Kundel, C. F. Nodine, E. F. Conant, and S. P. Weinstein, "Holistic component of image perception in mammogram interpretation: Gaze-tracking study," $R a$ diology, vol. 242, no. 2, pp. 396-402, 2007.

[17] T. Drew, K. Evans, M. L. Võ, F. L. Jacobson, and J. M. Wolfe, "Informatics in radiology: What can you see in a single glance and how might this guide visual search in medical images?," RadioGraphics, vol. 33, no. 1, pp. 263-274, 2013.

[18] J. Najemnik and W. S. Geisler, "Optimal eye movement strategies in visual search," Nature, vol. 434, no. 7031, pp. 387-391, 2005.

[19] J. Najemnik and W. S. Geisler, "Simple summation rule for optimal fixation selection in visual search," Vision Research, vol. 49, no. 10, pp. 1286-1294, 2009. 